\title{
Major considerations on the botulinum toxin in orofacial pain and tension headache: Systematic review
}

\author{
Priscilla Janaína de Lima Borelli Bovo ${ }^{1}$, Idiberto José Zotarelli Filho ${ }^{2 *}$ and Marcus Aurelius Nery Celino de Souza ${ }^{1}$ \\ ${ }^{1}$ Faculty of Western Center Paulista - Facop, Distrito Industrial de Piratininga/SP - 17490-000, São Paulo, Brazil \\ ${ }^{2}$ Post Graduate and Continuing Education (Unipos), Department of scientific production, Street Ipiranga, 3460, São José do Rio Preto SP, Brazil
}

\begin{abstract}
Introduction: Recent studies systematically show that there is scientific evidence on the efficacy of botulinum toxin type A (BTX-A) and B (BTX-B) in the treatment of myofascial pain related to masticatory muscles and consequent tension headache. However, more accurate and quantitative studies and forms of evaluation are needed for a definitive response on the efficacy and safety of this treatment.

Objective: to present and discuss, based on the main literary findings, the influence of the use of botulinum toxin on the reduction of orofacial pain and tension headache, in order to better understand the mechanisms of action and the real efficacy of this treatment.

Methods: Following the criteria of literary search with the use of the Mesh Terms that were cited in the item below on "Search strategies", the total of 76 papers that were submitted to the eligibility analysis were collated and, after that, 22 studies were selected, following the rules of systematic review-PRISMA. In general, as an example, the search strategy in MEDLINE / Pubmed, Web of Science, ScienceDirect Journals (Elsevier), Scopus (Elsevier), OneFile (Gale).

Major findings: BTX-B and BTX-A are associated with inhibition of the release of neuropeptides responsible for neurotransmission and / or peripheral and central sensitization of the pain pathway, as well as the reduction of the stimulated release of the peptide related to the calcitonin gene in cultures of trigeminal ganglion neurons. Furthermore, prior and subcutaneous administration of BTX-B-A in the rat paw allowed a significant reduction of the inflammatory pain induced. In addition, reduced activity of the dorsal horn neurons of the spinal cord was also observed.
\end{abstract}

Conclusion: Based on the literature findings, botulinum toxin type A or B therapy has been shown to be safe and effective in chronic pain disorders.

\section{Introduction}

Recent studies systematically show that there is scientific evidence on the efficacy of botulinum toxin type A (BTX-A) and B (BTX-B) in the treatment of myofascial pain related to masticatory muscles and consequent tension headache [1-4]. Research on the use of botulinum toxin for myofascial pain is contributing to improve treatments to date for this clinical condition [2]. Myofascial pain is due to regional neuromuscular alteration characterized by the presence of muscular contracture and consequently pain in the musculature of the face [5-8]. On the other hand, myofascial pain, when present for a prolonged time, is the etiological factor responsible for the onset of headaches, tensiontype headache $[9,10]$.

For the treatment of this syndrome there are several therapeutic procedures that most often act as controllers of painful symptomatology, without, however, effectively treating the cause [11]. However, a greater number of studies and precise and quantitative evaluation methods for a definitive answer on the efficacy and safety of this treatment $[11,12]$.

In this context, one of the highlighted therapies is the application of botulinum toxin to contraction muscles [11]. The side effects of botulinum toxin as a treatment for tension headache and myofascial pain syndrome were studied. In view of the obtained results, it was verified that botulinum toxin does not constitute a therapy that effectively treats headache, only controls for a determined time; it was also observed to cause side effects, such as hyperactivity and muscular pain, due to the overload imposed on the other chewing muscles [11].
As an explanation of the above, botulinum toxins are highly specific proteases cleaving presynaptic Soluble NSF Attachment proteins (SNARE) proteins involved in the exocytosis process of synaptic vesicles at the nerve terminals [11]. Thus, the destruction of these presynaptic proteins is the basis for the action of these toxins on the release of neurotransmitters $[13,14]$.

In this sense, botulinum toxin B (BTX-B) is a neurotoxin that has high affinity for cholinergic synapses, causing a block in the release of acetylcholine from these nerve terminals without altering the neural conduction of electrical signals or the synthesis and storage of acetylcholine [1,2,15-17]. The muscular injection of BTX-B causes partial chemical denervation and decrease of the contracture, without causing complete paralysis. The local intramuscular application of a selected dilution of BTX-B is followed by rapid diffusion into the interstitial space, finding the motor nerve endings of the skeletal muscles [18].

The aim of this study was to present and discuss the influence of botulinum toxin use on the reduction of orofacial pain and tension

${ }^{\star}$ Correspondence to: Idiberto José Zotarelli Filho, Unipos - Post graduate and continuing education, Street Ipiranga, 3460, São José do Rio Preto SP, Brazil 15020-040, E-mail: scientificresearch@unipos.com.br

Key words: myofascial pain, botulinum toxin, botulinum toxin type A, botulinum toxin type B, tension headache

Received: July 18, 2018; Accepted: August 08, 2018; Published: August 10, 2018 
headache, based on the main literary findings, in order to better understand the mechanisms of action and the real efficacy of this treatment.

\section{Methods}

\section{Study design}

Following the criteria of literary search with the use of the Mesh Terms that were cited in the item below on "Search strategies", a total of 76 papers that were submitted to the eligibility analysis were collated and, after that, 22 studies were selected, following the rules of systematic review-PRISMA (Transparent reporting of systematic reviews and meta-analyses-http://www.prisma-statement.org/).

\section{Sources of information}

The review protocol was based on the criteria of literary search with the use of mesh terms in the main databases such as Pubmed, Medline, Bireme, EBSCO, Scielo, etc. All references are registered in EndNote by the site: http://www.myendnoteweb.com/EndNoteWeb. html? cat=myrefs \&.

\section{Search strategy}

In general, as an example, the search strategy in MEDLINE / Pubmed, Web Of Science, ScienceDirect Journals (Elsevier), Scopus (Elsevier), OneFile (Gale) followed the following steps: - search for mesh terms: myofascial pain, botulinum toxin, botulinum toxin type A, botulinum toxin type $B$, tension headache, use of the bouleanos "and" between mesh terms and "or" among historical findings.

\section{Flow chart}

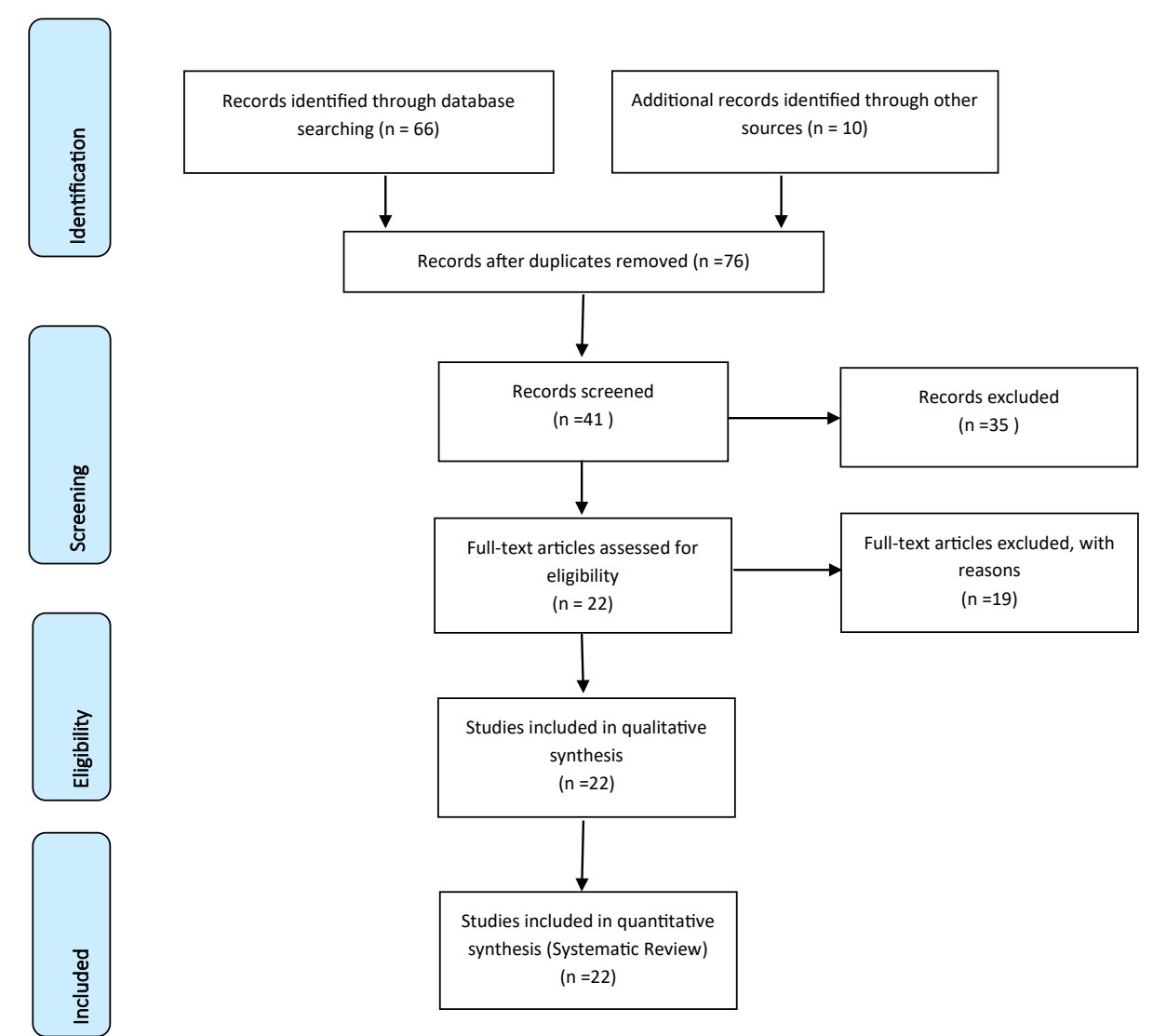

\section{Development and discussion}

\section{Mechanism of action - highlight}

The specificity of BTX-B for cholinergic nerves in vivo is due to the presence of specific receptors or acceptors in the motor nerve terminal membrane [18]. Furthermore, it has been observed that BTX-B can also inhibit norepinephrine exocytosis, its mechanism of action being identical to that observed in cholinergic synapses with SNARE complex cleavage [18-20]. However, in vivo, many nerve cells are deprived of the extracellular receptors / acceptors, responsible for the internalization of BTX-B, making it inefficient, since the only alternative entry route left in the nerve terminal is via the non-specific pinocitotic pathway [18].

In this context, botulinum toxins B and A (BTX-BA) were associated with the inhibition of the release of neuropeptides (glutamate, CGRP and substance $\mathrm{P}$ ) responsible for neurotransmission and / or peripheral and central sensitization of the pain pathway, as well as the reduction of stimulated release of the peptide related to the calcitonin gene (CGRP) in cultures of trigeminal ganglion neurons $[18,21]$. Furthermore, prior and subcutaneous administration of BTX-B-A in the rat paw allowed a significant reduction of the inflammatory pain induced. In addition, decreased activity of dorsal horn neurons of the spinal cord was also observed [18,21].

In addition to potent inhibitor of acetylcholine release, BTX-B may also have an inhibitory action on other neurotransmitters and neuropeptides, with anti-inflammatory and analgesic action [3,4]. Despite this, studies are necessary to better understand the mechanisms involved in the inhibitory action of BTX-B on the nocireceptor, but it is hypothesized that BTX-B can interrupt four painful signs: 1) Normalization of muscular hyperactivity; 2) Normalization of excessive 
muscle spindle activity; 3) Retrograde neuronal flow to the CNS; 4) Inhibition of neuropeptide release by the nociceptor, both in the peripheral tissue and in the central nervous system [4].

Thus, the inhibition of BTX-B release of acetylcholine occurs in a multifactorial manner [5]. First, BTX-B binds to the presynaptic membrane receptors of the motor nerve terminal irreversibly. This specificity at the binding site guarantees BTX-B high selectivity for cholinergic synapses. These presynaptic receptors are responsible for endocytosis of the neurotoxin to the motor nerve terminal $[5,6]$. After internalization of the molecule, it is separated into two polypeptide chains by proteases present in the motor nerve terminal. The cleavage of BTX-B is decisive for its activation. Thus, there are two fragments of polypeptides: one heavy chain with $100 \mathrm{kD}$ and the other with $50 \mathrm{kD}$ [6].

In this context, the percentage of cleavage is related to the serotype [7]. Serotype A has the highest percentage of cleavage, often 90.0 to $95.0 \%$, while serotype B is cleaved to a lesser extent, $70.0 \%$. The light chain is translocated through the endocytic vesicle membrane into the cytosol and binds with high specificity to the SNARE protein complex. The protein target also varies according to the BTX-B serotype [7]. Thus, the light chains of each of the seven serotypes cleave a distinct peptide bond at one or more points of the SNARE proteins, and none of the serotypes acts at the same site [7].

Thus, the obvious clinical action is dose-dependent weakness or paralysis in skeletal muscle at motor nerve endings [8]. The first effect on the musculature is on the function of the alpha motor neuron, responsible for the stimulation of the muscle fibers themselves, but BTX-B can also affect the gamma motor neurons that innervate the muscle spindle [8]. Inhibition of the latter results in decreased muscle tone, due to the consequent decrease of the afferent feedback on the alpha motor neuron, originating from the muscle spindle [8].

\section{Major literary findings}

In a systematic review study with six literary studies, the primary outcomes were initial and post-treatment pain intensity and patients who reported pain relief of $50.0 \%$ or more after using BTX-A [1]. For a dichotomous outcome of pain reduction of $50.0 \%$ or more, relative risk (risk ratio) with 95\% CI was reported. Statistical heterogeneity was tested with the Cochran test for heterogeneity and quantified by statistics. The results were combined using the random effects model in the presence of statistical heterogeneity, otherwise, fixed-effect model was used [1]. The effects of the interventions were presented in three meta-analyzes. The mean difference in pooled posttreatment pain scores (six studies) showed a reduction of -3.009 in EVA (95\% CI -4.566 to $-1.453 \mathrm{p}<0.001)$ with the use of BTX-A. The standardized difference in mean post-treatment pain (six studies) was $-0.918(95 \%$ CI -1.197 to $-0.639 \mathrm{p}<0.001)$ in favor of BTX-A. For the percentage of patients with $50 \%$ pain reduction (three studies), the absolute risk and relative risk difference (RR 2,892, 95\% CI 1,726 to $4,848 \mathrm{p}<0.001$ ) was calculated in favor of BTX-A. Therefore, the authors concluded that there is moderate evidence on the efficacy of BTX-A in the treatment of patients with trigeminal neuralgia and post-herpetic neuralgia [1].

Another 2018 study has shown that the central antinociceptive effect of BTX-A in the sciatic region also involves the endogenous opioid system [2]. Thus, the influence of the central acting opioid antagonist naltrexone $(2 \mathrm{mg} / \mathrm{kg}$ ) versus peripheral action methylnaltrexone $(2 \mathrm{mg} / \mathrm{kg})$ on BTX-A (5 U / kg) was investigated in the orofacial formalin test, the antinociceptive effect of morphine $(6 \mathrm{mg} / \mathrm{kg})$ and the effect on neurogenic dural inflammation (DNI). DNI was assessed by extravasation of Evans blue plasma proteins. As a result, naltrexone abolished the effect of BTX-A on the extravasation of pain and dural plasma protein, whereas peripheral-acting methylnaltrexone did not alter the effect of BTX-A on pain or its effect on dura mater leakage [2]. Naltrexone also abolished the antinociceptive and anti-inflammatory effects of morphine. However, methylnaltrexone decreased the antinociceptive effect of morphine only partially in the second phase of the test and had no significant effect on the morphine-mediated reduction in DNI. Morphine acts on pain in the trigeminal region both peripherally and centrally, while the effect on dural plasma protein extravasation appears to be only centrally mediated. However, the interaction of BTX-A with the endogenous opioid system, with the consequent inhibition of nociceptive transmission, as well as DNI, occurs mainly at the center [2].

In this scenario, however, conventional treatments of myofascial pain syndrome are often unsatisfactory, with episodes persisting for at least one year, leading to nephropathies [3]. With BTX-B, in a single application, benefits can be obtained for one to three months when accompanied by adequate physiotherapy without the side effect of impaired renal function [3].

In a comparative study between dry needling, local anesthetic (lidocaine) in local injection and low doses of BTX-B in the treatment of trigger points in the myofascial pain syndrome, there was an increase in the range of movements in the cervical musculature in the three groups. As for pain, fatigue and disability for work, measured by the Visual Analogue Scale (VAS), there was an important decrease in the injection group with lidocaine and BTX-B, and the same result was not observed with dry needling [16].

However, lidocaine was shown to be more effective than BTX-B when compared to dry needling. Sensations such as fatigue, muscle pain and headache may be referred after BTX-B-A injection. However, these side effects last a few days. In addition, in TMD (temporomandibular disorder), caused by chronic myofascial pain,

BTX-B-A is clinically indicated. Myofascial pain results from hyperactivity of the masticatory musculature and hypermobility of the condyle and may radiate to the region of the affected muscle. Thus, muscles that close the mandible (masseter, temporal and medial pterygoid) and those that protrude the mandible (lateral pterygoid) are affected [16].

In a prospective, placebo-controlled study, 90 patients with TMD were previously treated with conservative treatment (myo-relaxant plaque and physiotherapy with relaxing techniques and massage) for a minimum of three and a maximum of 34 months [14]. Sixty of these patients received BTX-B-A 35UI and 30 received $\mathrm{NaCl}$ solution $(0.9 \%$ saline solution) in masseter, temporal and pterygoid lateral muscles via the oral route $(77.0 \%)$. Some muscles were approached by the extra oral route as temporal and masseter (23.0\%). Injections were given in the most painful regions of the muscle. The dilution of BTX-B-A was done with $0.7 \mathrm{~mL}$ of $0.9 \%$ physiological solution. Both the placebo (physiological) solution and the BTX-B-A solution were injected into the muscles bilaterally. Patients were asked to suspend other treatments for their pain seven days before the first injection [14]. The results showed improvement in 55 patients (91\%) in the BTX-B-A group with a decrease of 3.2 points in the visual analogue scale (VAS). In the placebo group, the local pain improvement was only 0.4 points in the VAS. Patients with more severe pain (VAS greater than 6.5 ) showed significant improvement [14]. 
In this sense, the dose of BTX-B-A used was relatively low, particularly in the study by Silberstein et al. (2006) [17] with 25 IU of BTX-B-A. The variation of results in clinical studies with BTXB-A could have several origins, such as patient selection, protocol of injections and dose variation used, concomitant use of prophylactic medication for headache. More frequent adverse effects, possible to occur are muscle weakness, cervical stiffness, cervical pain, and this occurrence is approximately $3.0 \%$ [17]. Positive results in headache treatment may appear 90 days after application [17].

BTX-B-A is effective in the prophylaxis of various types of headache, including migraine. Thus, headaches with muscle disorders respond to treatment with BTX-B-A, including cervicogenic headache and chronic headache associated with a whiplike cervical lesion [13]. In Migraine, the mechanism of action of BTX-B-A is believed to be by relaxing the muscles infiltrated by BTX-B-A and also, consequently, decreasing the pressure on the roots of the trigeminal nerve [13].

\section{Conclusion}

Based on the literary findings, therapy with botulinum toxin type A or B has been shown to be safe and effective in chronic pain disorders. Also, there was reduction of adjuvant medications and time of action, lasting from three to four months per dose.

\section{Declaration of potential conflict of interest}

The authors declare no conflict of interest.

\section{References}

1. Fischoff DK, Spivakovsky S1 (2018) Botulinum toxin for facial neuralgia. Evid Based Dent 19: 57-58. [Crossref]

2. Drinovac Vlah V, Filipovic B, Bach-Rojecky L, Lackovic Z (2018) Role of central versus peripheral opioid system in antinociceptive and anti-inflammatory effect of botulinum toxin type A in trigeminal region. Eur J Pain 22: 583-591. [Crossref]

3. Drinovac Vlah V, Bach-Rojecky L, Lackovic Z (2016) Antinociceptive action of botulinum toxin type A in carrageenan-induced mirror pain. J Neural Transm (Vienna) 123: 1403-1413. [Crossref]

4. Lackovic Z, Filipovic B, Matak I, Helyes Z (2016) Activity of botulinum toxin type A in cranial dura: implications for treatment of migraine and other headaches. $\mathrm{Br} J$ Pharmacol 173: 279-291. [Crossref]

5. Drinovac V, Bach-Rojecky L, Matak I, Lackovic Z (2013) Involvement of opioid receptors in antinociceptive action of botulinum toxin type A. Neuropharmacology 70 : 331-337. [Crossref]
6. Drinovac V, Bach-Rojecky L, Lackovic Z (2014) Association of antinociceptive action of botulinum toxin type A with GABA-A receptor. J Neural Transm (Vienna) 121: 665669. [Crossref]

7. Matak I, Rossetto O, Lackovic Z (2014) Botulinum toxin type A selectivity for certain types of pain is associated with capsaicin-sensitive neurons. Pain 155: 1516-1526. [Crossref]

8. Antonucci F, Rossi C, Gianfranceschi L, Rossetto O, Caleo M (2008) Long-distance retrograde effects of botulinum neurotoxin A. J Neurosci 28: 3689-3696. [Crossref]

9. Bach-Rojecky L, Lackovic Z (2009) Central origin of the antinociceptive action of botulinum toxin type A. Pharmacol Biochem Behav 94: 234-238. [Crossref]

10. Bach-Rojecky L, Relja M, Lackovic Z (2005) Botulinum toxin type A in experimental neuropathic pain. J Neural Transm (Vienna) 112: 215-219. [Crossref]

11. Raulino NB, Mauro M, Bento AB, Hugo CJ, Ana RBRS, Isabella SSH (2013) Effect of botulinum toxin in the treatment of tension type headache. Rev Odontol Bras Central 21.

12. Carrara SV, Cont PCR, Barbosa JS (2010) Termo do $1^{\circ}$ Consenso em Disfunção temporomandibular e Dor Orofacial. Dental Press J Orthod 15: 1140.

13. Gazerani P, Pederson NS, Staahl C, Drewes AM, Arendt-Nielsen L (2009) Subcutaneous botulinum toxin type a reduces capsaicin-induced trigeminal pain and vasomotor reactions in human skin. Pain 141: 60-69.

14. von Lindern JJ, Niederhagen B, Bergé S, Appel T (2003) Type A botulinum toxin in the treatment of chronic facial pain associated with masticatory hyperactivity. $J$ Oral Maxillofac Surg 61: 774-778. [Crossref]

15. Jabbari B, Ney J, Sichani A, Monacci W, Foster L, et al. (2006) Treatment of refractory, chronic low back pain with botulinum neurotoxin A: an open-label, pilot study. Pain Med 7: 260-264. [Crossref]

16. Gassner HG, Sherris DA (2000) Addition of an anesthetic agent to enhance the predictability of the effects of botulinum toxin type A injections: a randomized controlled study. Mayo Clin Proc 75: 701-704. [Crossref]

17. Silberstein SD, Göbel H, Jensen R, Elkind AH, Degryse R, et al. (2006) Botulinum toxin type A in the prophylactic treatment of chronic tension-type headache: a multicentre, double-blind, randomized, placebo-controlled, parallel-group study. Cephalalgia 26: 790-800. [Crossref]

18. Smuts JA, Schultz D, Barnard A (2004) Mechanism of action of botulinum toxin type A in migraine prevention: a pilot study. Headache 44: 801-805. [Crossref]

19. Allen G, Galer BS, Schwartz L (1999) Epidemiology of complex regional pain syndrome: a retrospective chart review of 134 patients. Pain 80: 539-544. [Crossref]

20. Argoff CE (1999) Botulinum toxin type A treatment of myofascial pain with CRPS type I (reflex sympathetic dystrophy): a pilot study, em: World Pain Congress (IASP), Vienna, Austria.

21. Jabbari B, Maher N (2002) Effectiveness of botulinum toxin A against the segmental burning pain of spinal cord origin. In: International Conference 2002: Basic and Therapeutic Aspects of Botulinum and Tetanus Toxins, Hannover, Germany.

22. Lang AM (2003) Botulinum toxin type A therapy in chronic pain disorders. Arch Phys Med Rehabil 84: S69-73. [Crossref]

Copyright: (C2018 de Lima Borelli Bovo PJ. This is an open-access article distributed under the terms of the Creative Commons Attribution License, which permits unrestricted use, distribution, and reproduction in any medium, provided the original author and source are credited. 